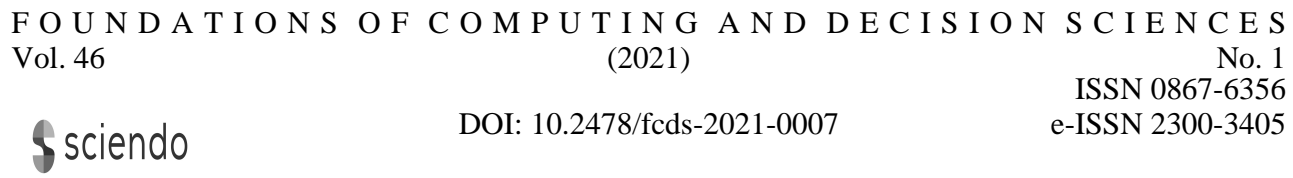

\title{
Investigation of E-Cigarette Smoking Model with Mittag-Leffler Kernel
}

\author{
Esmehan Uçar *, Sümeyra Uçar, Fırat Evirgen, Necati Özdemir ${ }^{\dagger}$
}

\begin{abstract}
Smoking is the most lethal social poisoning event. The World Health Organization defines smoking as the most important preventable cause of disease. Around 4.9 million people worldwide die from smoking every year. In order to analysis this matter, we aim to investigate an e-cigarette smoking model with AtanganaBaleanu fractional derivative. We obtain the existence conditions of the solution for this fractional model utilizing fixed-point theory. After giving existence conditions, the uniqueness of the solution is proved. Finally, to show the effect of the AtanganaBaleanu fractional derivative on the model, we give some numerical results supported by illustrative graphics.
\end{abstract}

Keywords: Atangana-Baleanu derivative, fixed point theory, e-cigarette smoking model, numerical method.

\section{Introduction}

Smoking is a major public health problem in the world and has the potential to be addictive because it contains a high proportion of nicotine. Tobacco products contain more than 4000 chemicals. In individuals many diseases and disturbances like cardiovascular diseases, lung diseases, Chronic obstructive pulmonary disease (COPD), stomach cancer, skin cancer, etc. can be seen by the effect of this additive material [22].

For this purpose, The World Health Organization (WHO) took the first step in 2003 with the Framework Convention on Tobacco Control (WHO-FCTC) to fight with cigarette consumption worldwide. Then, in 2008, the WHO introduced the MPOWER measures in line with WHO-FCTC. If WHO-FCTC measures represented

\footnotetext{
*Department of Mathematics, Balıkesir University, Turkey, esucarr@gmail.com

${ }^{\dagger}$ Department of Mathematics, Faculty of Arts and Sciences, Balıkesir University, Turkey, \{sumeyraucar, fevirgen, nozdemir\}@balikesir.edu.tr
} 
by MPOWER are fully implemented, smoking prevalence in the Eastern Mediterranean Region is expected to result in a 10\% reduction in 2030 [23].

However, a different danger in the sense of smoking habit has started to become widespread among young people. This danger is e-cigarettes and that known in the literature as electronic nicotine delivery systems (ENDS) or electronic non-nicotine delivery systems (ENNDS). The perception that it is less harmful than traditional cigarettes is the main reason why these products are so popular among young people. However, many studies and reports have shown that, contrary to this perception, these products also contain nicotine and other toxic substances, which are typically harmful to both consumers and those exposed to aerosols second hand [22, 5]. Furthermore, the studies in the recent year show that the consumption of e-cigarette cause suicidal behavior on young people $[11,6]$.

Mathematical modeling comes to the fore as an important tool to protect human health against such dangers and diseases. Many mathematical models are discussed in the literature on various topics. In 1993 Perelson et al. [15] examined a model for the interaction of HIV with $C D 4+T$ cells. Yadav et al. [24] analyzed the mathematical model to study the dynamics of smoking behavior under the influence of educational programs. Shabestari et al. [16] proposed a new model for the interaction between insulin and glucose.

Therewithal, the concept of fractional analysis has recently played a major role in the construction of these models and to study of their effects. Many researchers have contributed to the literature with their studies showing that the fractional models express even the real structure better than the classical models. Atangana and Koca 2016 [2] explored new behavior of the chaotic system by using the Atangana-Baleanu fractional derivative. In 2018 Koca $[12,13]$ analyzed the effect of the AtanganaBaleanu fractional operators on the Ebola virus and rubella disease. In 2018 Jajarmi and Baleanu [8] investigated the pathological behavior of HIV-infection using a new model in fractional calculus. Singh et al. [17] proved the existence and uniqueness of the solution of a fractional epidemiological model for computer viruses. Ucar et al. [21] investigated a fractional glucose-insulin regulatory system with non-singular kernel derivative. Kumar et al. [14] studied an efficient numerical scheme for the fractional model of HIV infection. Evirgen et al. [3] investigated the effect of non-singular kernel derivative on the HIV infection model with $C D 4+T$. Yavuz and Yokus [26] explained a nerve impulse model in the sense of conformable operator. Yavuz and Özdemir [25] considered the SIR model using the Caputo-Fabrizio fractional derivative to obtain the special solutions.

From the mathematical modeling point of view, smoking cessation models have been generally studied by using mathematical modeling. Using integer order derivative, Zaman et al. [28] used optimal control theory to minimize the number of light and persistent smokers and maximize the number of quit smokers in a community, Khalid et al. [10] give perturbation-iteration algorithm for fractional giving up smoking mathematical model. Moreover, Uçar et al. [20] consider a smoking model with fractional Atangana-Baleanu derivative. On the one hand, there is a limited number of e-cigarette smoking models in the literature. Hung et al. [7] established a mathematical model for smoking cessation with e-cigarettes and presented the theoretical 
study for the effect of e-cigarettes, Straughan [18] considered a differential equation model for the effect smoking e-cigarettes may have upon smokers of tobacco cigarettes or on non-smokers. To the best of the authors' knowledge, there are only these two studies about e-cigarette smoking in the literature. With these motivations, in this study we aim to discuss the mathematical model developed by Straughan [18] in 2018 and expressing the habit of e-cigarettes under peer pressure. First, we enlarge this model benefiting from Atangana-Baleanu fractional derivative [1]. The existence and the uniqueness of the solutions of the fractional model are theoretically proved. Also, the effect of fractional derivative on the model is numerically examined and expressed in graphics with the arbitrary order derivative.

\section{Some preliminaries}

In this section, we give basic definitions of the $\mathrm{AB}$ fractional derivative.

Definition 1 Let $g \in H^{1}(a, b), a<b, \sigma \in[0,1]$, the Atangana-Baleanu derivative in Caputo type is given by

$$
{ }_{a}^{A B C} D_{t}^{\sigma}[g(t)]=\frac{L(\sigma)}{1-\sigma} \int_{a}^{t} g^{\prime}(x) E_{\sigma}\left[-\sigma \frac{(t-x)^{\sigma}}{1-\sigma}\right] d x
$$

where $t$ corresponds time, $x$ is integral variable and $L(\sigma)$ is a normalization function with $L(0)=L(1)=1[1]$.

Definition 2 Let $g \in H^{1}(a, b), a<b, \sigma \in[0,1]$, the Atangana-Baleanu derivative in Riemann-Liouville type is given by [1]:

$$
{ }_{a}^{A B R} D_{t}^{\sigma}[g(t)]=\frac{L(\sigma)}{1-\sigma} \frac{d}{d t} \int_{a}^{t} g(x) E_{\sigma}\left[-\sigma \frac{(t-x)^{\sigma}}{1-\sigma}\right] d x .
$$

Definition 3 The fractional integral related to the fractional derivative is defined by [1]:

$$
{ }_{a}^{A B} I_{t}^{\sigma}[g(t)]=\frac{1-\sigma}{L(\sigma)} g(t)+\frac{\sigma}{L(\sigma) \Gamma(\sigma)} \int_{a}^{t} g(\lambda)(t-\lambda)^{\sigma-1} d \lambda .
$$




\section{E-cigarette smoking model with fractional derivative}

In this section, we will investigate the e-cigarette smoking model discusses in the below expression [18]:

$$
\begin{aligned}
\frac{d C}{d t} & =\mu N-\mu C-\beta C \frac{S}{N} \\
\frac{d S}{d t} & =\beta C \frac{S}{N}+\alpha E-\mu S-\gamma_{1} S \frac{E}{N}, \\
\frac{d E}{d t} & =-\alpha E-\mu E+\gamma_{1} S \frac{E}{N} .
\end{aligned}
$$

Because $N=C+S+E$ is constant, if we take $c=\frac{C}{N}, s=\frac{S}{N}, e=\frac{E}{N}$ in the (4), we get

$$
\begin{aligned}
& \frac{d c}{d t}=\mu-\mu c-\beta c s \\
& \frac{d s}{d t}=\beta c s+\alpha e-\mu s-\gamma_{1} s e \\
& \frac{d e}{d t}=-(\alpha+\mu) e+\gamma_{1} s e .
\end{aligned}
$$

with the initial conditions $c(0), s(0), e(0)$. The model parameters are given in Table 1 and the transmission between the model parameters are visualized by Figure 1.

Table 1. State variables and parameters of the e-cigarette smoking model (4).

\begin{tabular}{cl}
\hline Parameter & Description \\
\hline$C(t)$ & non-smoker class \\
$S(t)$ & smokers of tobacco cigaretttes \\
$E(t)$ & smokers of e-cigarettes \\
$\alpha$ & those whosmoke e-cigarettes returning to smoking cigarettes \\
$\beta$ & a ratio which shows the probability of a non-smoker becoming a smoker \\
$\gamma_{1}$ & a ratio which displays the probability of switching fromsmoking to smoking e-cigarettes \\
$\mu$ & a leaving or death ratio \\
\hline
\end{tabular}

Now, we modify this model by the AB derivative in Caputo type:

$$
\begin{aligned}
& { }_{0}^{A B C} D_{t}^{\sigma} c(t)=\mu-\mu c-\beta c s, \\
& { }_{0}^{A B C} D_{t}^{\sigma} s(t)=\beta c s+\alpha e-\mu s-\gamma_{1} s e, \\
& { }_{0}^{A B C} D_{t}^{\sigma} e(t)=-(\alpha+\mu) e+\gamma_{1} s e .
\end{aligned}
$$

with the above same initial conditions and where ${ }_{0}^{A B C} D_{t}^{\sigma}$ is $\mathrm{AB}$ derivative in Caputo type. 


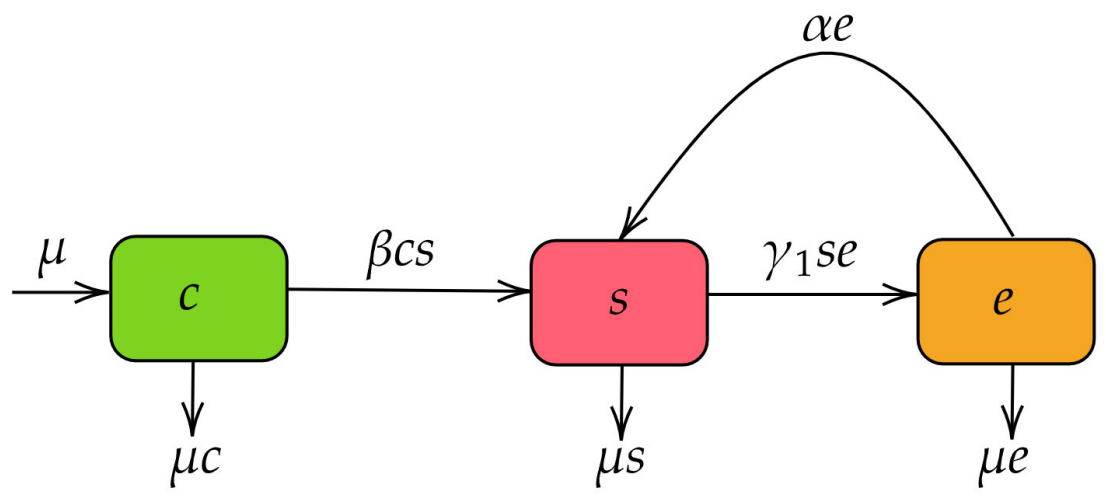

Figure 1. The transfer diagram of the e-cigarette smoking model (5).

By taking related integral, the above system is convert to the followings:

$$
\begin{aligned}
& c(t)-\widetilde{k}_{1}(t)=\frac{1-\sigma}{M(\sigma)}\{\mu-\mu c(t)-\beta c(t) s(t)\} \\
& +\frac{\sigma}{M(\sigma) \Gamma(\sigma)} \int_{0}^{t}(t-\lambda)^{\sigma-1}\{\mu-\mu c(\lambda)-\beta c(\lambda) s(\lambda)\} d \lambda \\
& s(t)-\widetilde{k}_{2}(t)=\frac{1-\sigma}{M(\sigma)}\left\{\beta c(t) s(t)+\alpha e(t)-\mu s(t)-\gamma_{1} s(t) e(t)\right\} \\
& +\frac{\sigma}{M(\sigma) \Gamma(\sigma)} \int_{0}^{t}(t-\lambda)^{\sigma-1}\left\{\beta c(\lambda) s(\lambda)+\alpha e(\lambda)-\mu s(\lambda)-\gamma_{1} s(\lambda) e(\lambda)\right\} d \lambda, \\
& e(t)-\widetilde{k}_{3}(t)=\frac{1-\sigma}{M(\sigma)}\left\{-(\alpha+\mu) e(t)+\gamma_{1} s(t) e(t)\right\} \\
& +\frac{\sigma}{M(\sigma) \Gamma(\sigma)} \int_{0}^{t}(t-\lambda)^{\sigma-1}\left\{-(\alpha+\mu) e(\lambda)+\gamma_{1} s(\lambda) e(\lambda)\right\} d \lambda .
\end{aligned}
$$

where $\widetilde{k}_{1}, \widetilde{k}_{2}, \widetilde{k}_{3}$ are constants. Iteratively, the above can be given by

$$
\begin{aligned}
& c_{0}(t)=\widetilde{k}_{1}(t), \\
& s_{0}(t)=\widetilde{k}_{2}(t), \\
& e_{0}(t)=\widetilde{k}_{3}(t),
\end{aligned}
$$


and

$$
\begin{aligned}
& c_{n+1}(t)=\frac{1-\sigma}{M(\sigma)}\left\{\mu-\mu c_{n}(t)-\beta c_{n}(t) s_{n}(t)\right\} \\
& +\frac{\sigma}{M(\sigma) \Gamma(\sigma)} \int_{0}^{t}(t-\lambda)^{\sigma-1}\left\{\mu-\mu c_{n}(\lambda)-\beta c_{n}(\lambda) s_{n}(\lambda)\right\} d \lambda \\
& s_{n+1}(t)=\frac{1-\sigma}{M(\sigma)}\left\{\beta c_{n}(t) s_{n}(t)+\alpha e_{n}(t)-\mu s_{n}(t)-\gamma_{1} s_{n}(t) e_{n}(t)\right\} \\
& +\frac{\sigma}{M(\sigma) \Gamma(\sigma)} \int_{0}^{t}(t-\lambda)^{\sigma-1}\left\{\beta c_{n}(\lambda) s_{n}(\lambda)+\alpha e_{n}(\lambda)-\mu s_{n}(\lambda)-\gamma_{1} s_{n}(\lambda) e_{n}(\lambda)\right\} d \lambda \\
& e_{n+1}(t)=\frac{1-\sigma}{M(\sigma)}\left\{-(\alpha+\mu) e_{n}(t)+\gamma_{1} s_{n}(t) e_{n}(t)\right\} \\
& +\frac{\sigma}{M(\sigma) \Gamma(\sigma)} \int_{0}^{t}(t-\lambda)^{\sigma-1}\left\{-(\alpha+\mu) e_{n}(\lambda)+\gamma_{1} s_{n}(\lambda) e_{n}(\lambda)\right\} d \lambda
\end{aligned}
$$

We expect to obtain the exact solution, if we take the limit for a large value of $n$.

\section{Using Picard-Lindelof approach to obtain the uniqueness of solutions}

Here, we define the operator below:

$$
\begin{aligned}
& h_{1}(t, c)=\mu-\mu c(t)-\beta c(t) s(t), \\
& h_{2}(t, s)=\beta c(t) s(t)+\alpha e(t)-\mu s(t)-\gamma_{1} s(t) e(t), \\
& h_{3}(t, e)=-(\alpha+\mu) e(t)+\gamma_{1} s(t) e(t),
\end{aligned}
$$

where $h_{1}, h_{2}, h_{3}$ are contraction with respect to the functions $c, s$ and $e$. Let

$$
\begin{aligned}
& M_{1}=\sup _{C\left[a, b_{1}\right]}\left\|h_{1}(t, c)\right\|, \\
& M_{2}=\sup _{C\left[a, b_{2}\right]}\left\|h_{2}(t, s)\right\|, \\
& M_{3}=\sup _{C\left[a, b_{3}\right]}\left\|h_{3}(t, e)\right\|,
\end{aligned}
$$

where

$$
\begin{aligned}
& C\left[a, b_{1}\right]=[t-a, t+a] \times\left[x-b_{1}, x+b_{1}\right]=A \times B_{1}, \\
& C\left[a, b_{2}\right]=[t-a, t+a] \times\left[x-b_{2}, x+b_{2}\right]=A \times B_{2}, \\
& C\left[a, b_{3}\right]=[t-a, t+a] \times\left[x-b_{3}, x+b_{3}\right]=A \times B_{3} .
\end{aligned}
$$


We apply Banach-fixed point theorem benefiting from the metric on $C\left[a, b_{i}\right](i=1,2,3)$ with the norm

$$
\|f(t)\|=\sup _{t \in[t-a, t+a]}|f(t)| .
$$

Considering the Picard's operator, we find

$$
\Theta: C\left(A, B_{1}, B_{2}, B_{3}\right) \rightarrow C\left(A, B_{1}, B_{2}, B_{3}\right)
$$

defined as

$$
\Theta X(t)=X_{0}(t)+X(t) \frac{1-\sigma}{M(\sigma)}+\frac{\sigma}{M(\sigma) \Gamma(\sigma)} \int_{0}^{t}(t-\lambda)^{\sigma-1} F(\lambda, X(\lambda)) d \lambda
$$

where

$$
\begin{aligned}
X(t) & =\left[\begin{array}{lll}
c(t) & s(t) & e(t)
\end{array}\right]^{T}, \\
X_{0}(t) & =\left[\begin{array}{lll}
c(0) & s(0) & e(0)
\end{array}\right]^{T}, \\
F(t, X(t)) & =\left[\begin{array}{lll}
k_{1}(t, c(t)) & k_{2}(t, s(t)) & k_{3}(t, e(t))
\end{array}\right]^{T} .
\end{aligned}
$$

Assume that the following inequality satisfied

$$
\|X(t)\|_{\infty} \leq \max \left\{b_{1}, b_{2}, b_{3}\right\} .
$$

We take into consideration

$$
\begin{aligned}
\left\|\Theta X(t)-X_{0}(t)\right\| & =\left\|\frac{1-\sigma}{M(\sigma)} F(t, X(t))+\frac{\sigma}{M(\sigma) \Gamma(\sigma)} \int_{0}^{t}(t-\lambda)^{\sigma-1} F(\lambda, X(\lambda)) d \lambda\right\| \\
& \leq \frac{1-\sigma}{M(\sigma)}\|F(t, X(t))\|+\frac{\sigma}{M(\sigma) \Gamma(\sigma)} \int_{0}^{t}(t-\lambda)^{\sigma-1}\|F(\lambda, X(\lambda))\| d \lambda \\
& \leq \frac{1-\sigma}{M(\sigma)} M+\frac{\sigma}{M(\sigma) \Gamma(\sigma)} M a^{\sigma}
\end{aligned}
$$

where $M=\max \left\{M_{1}, M_{2}, M_{3}\right\}$. Let $b=\max \left\{b_{1}, b_{2}, b_{3}\right\}$ and $a<\frac{b}{M}$. So we have $\left\|\Theta X(t)-X_{0}(t)\right\|<a M<b$. Also we have the following

$$
\left\|\Theta X_{1}-\Theta X_{2}\right\|=\sup _{t \in A}\left|X_{1}-X_{2}\right|
$$


Then, we have

$$
\begin{aligned}
\left\|\Theta X_{1}-\Theta X_{2}\right\| & =\| \frac{1-\sigma}{M(\sigma)}\left(F\left(t, X_{1}(t)\right)-F\left(t, X_{2}(t)\right)\right) \\
& +\frac{\sigma}{M(\sigma) \Gamma(\sigma)} \int_{0}^{t}(t-\lambda)^{\sigma-1}\left(F\left(\lambda, X_{1}(\lambda)\right)-F\left(\lambda, X_{2}(\lambda)\right)\right) d \lambda \| \\
& \leq \frac{1-\sigma}{M(\sigma)} q\left\|X_{1}(t)-X_{2}(t)\right\| \\
& +\frac{\sigma q}{M(\sigma) \Gamma(\sigma)} \int_{0}^{t}(t-\lambda)^{\sigma-1}\left\|X_{1}(\lambda)-X_{2}(\lambda)\right\| d \lambda \\
& \leq\left(\frac{1-\sigma}{M(\sigma)} q+\frac{\sigma q a^{q}}{M(\sigma) \Gamma(\sigma)}\right)\left\|X_{1}(t)-X_{2}(t)\right\| \\
& \leq a q\left\|X_{1}(t)-X_{2}(t)\right\|
\end{aligned}
$$

with $q<1$. Because the function operator $\mathrm{F}$ is contraction, we get $a q<1$, and $\Theta$ is contraction. Thus, the system (6) has a unique set of solution, and also exists.

\section{Numerical method for the fractional e-cigarette smoking model}

In order to get solution of some problems defined by fractional derivatives with nonlocal and nonsingular kernel, Toufik and Atangana present a new numerical method based on two-step Lagrange polynomial technique, also their method converges rapidly to the exact solution are showed by these researchers [19].

Now, we handle our fractional e-cigarette smoking model (6). Applying AB fractional integral to both sides, we get

$$
\begin{aligned}
& c(t)-c(0)=\frac{1-\sigma}{M(\sigma)} h_{1}(t, c(t))+\frac{\sigma}{M(\sigma) \Gamma(\sigma)} \int_{0}^{t} h_{1}(\lambda, c(\lambda))(t-\lambda)^{\sigma-1} d \lambda, \\
& s(t)-s(0)=\frac{1-\sigma}{M(\sigma)} h_{2}(t, s(t))+\frac{\sigma}{M(\sigma) \Gamma(\sigma)} \int_{0}^{t} h_{2}(\lambda, s(\lambda))(t-\lambda)^{\sigma-1} d \lambda, \\
& e(t)-e(0)=\frac{1-\sigma}{M(\sigma)} h_{3}(t, e(t))+\frac{\sigma}{M(\sigma) \Gamma(\sigma)} \int_{0}^{t} h_{3}(\lambda, e(\lambda))(t-\lambda)^{\sigma-1} d \lambda,
\end{aligned}
$$

with the initial conditions $c(0)=c_{0}, s(0)=s_{0}, e(0)=e_{0}$. At a point $t=t_{n+1}$, we 
handle the so-called numerical method for the above system.

$$
\begin{aligned}
& c_{n+1}=c_{0}+\frac{1-\sigma}{M(\sigma)} h_{1}\left(t_{n}, c\left(t_{n}\right)\right) \\
& +\frac{\sigma}{M(\sigma)} \sum_{k=0}^{n}\left(\frac{h^{\sigma} h_{1}\left(t_{k}, c_{k}\right)}{\Gamma(\sigma+2)}\left((n-k+1)^{\sigma}(n-k+2+\sigma)-(n-k)^{\sigma}(n-k+2+2 \sigma)\right)\right. \\
& \left.-\frac{h^{\sigma} h_{1}\left(t_{k-1}, c_{k-1}\right)}{\Gamma(\sigma+2)}\left((n-k+1)^{\sigma+1}-(n-k)^{\sigma}(n-k+1+\sigma)\right)\right)+{ }^{1} R_{n}^{\sigma}, \\
& s_{n+1}=s_{0}+\frac{1-\sigma}{M(\sigma)} h_{2}\left(t_{n}, s\left(t_{n}\right)\right) \\
& +\frac{\sigma}{M(\sigma)} \sum_{k=0}^{n}\left(\frac{h^{\sigma} h_{2}\left(t_{k}, s_{k}\right)}{\Gamma(\sigma+2)}\left((n-k+1)^{\sigma}(n-k+2+\sigma)-(n-k)^{\sigma}(n-k+2+2 \sigma)\right)\right. \\
& \left.-\frac{h^{\sigma} h_{2}\left(t_{k-1}, s_{k-1}\right)}{\Gamma(\sigma+2)}\left((n-k+1)^{\sigma+1}-(n-k)^{\sigma}(n-k+1+\sigma)\right)\right)+{ }^{2} R_{n}^{\sigma}, \\
& e_{n+1}=e_{0}+\frac{1-\sigma}{M(\sigma)} h_{3}\left(t_{n}, e\left(t_{n}\right)\right) \\
& +\frac{\sigma}{M(\sigma)} \sum_{k=0}^{n}\left(\frac{h^{\sigma} h_{3}\left(t_{k}, e_{k}\right)}{\Gamma(\sigma+2)}\left((n-k+1)^{\sigma}(n-k+2+\sigma)-(n-k)^{\sigma}(n-k+2+2 \sigma)\right)\right. \\
& \left.-\frac{h^{\sigma} h_{3}\left(t_{k-1}, e_{k-1}\right)}{\Gamma(\sigma+2)}\left((n-k+1)^{\sigma+1}-(n-k)^{\sigma}(n-k+1+\sigma)\right)\right)+{ }^{3} R_{n}^{\sigma},
\end{aligned}
$$

where ${ }^{k} R_{n}^{\sigma}, j=1,2,3$ are remainder terms of the form:

$$
\begin{aligned}
{ }^{1} R_{n}^{\sigma} & =\frac{\sigma}{M(\sigma) \Gamma(\sigma)} \sum_{k=0}^{n} \int_{t_{k}}^{t_{k-1}} \frac{\left(\lambda-t_{k}\right)\left(\lambda-t_{k-1}\right)}{2 !} \frac{\partial^{2}}{\partial \lambda^{2}}\left[h_{1}(\lambda, c(\lambda))\right]_{y=\epsilon_{y}}\left(t_{n+1}-\lambda\right)^{\sigma-1} d \lambda, \\
{ }^{2} R_{n}^{\sigma} & =\frac{\sigma}{M(\sigma) \Gamma(\sigma)} \sum_{k=0}^{n} \int_{t_{k}}^{t_{k-1}} \frac{\left(\lambda-t_{k}\right)\left(\lambda-t_{k-1}\right)}{2 !} \frac{\partial^{2}}{\partial \lambda^{2}}\left[h_{2}(\lambda, s(\lambda))\right]_{y=\epsilon_{y}}\left(t_{n+1}-\lambda\right)^{\sigma-1} d \lambda, \\
{ }^{3} R_{n}^{\sigma} & =\frac{\sigma}{M(\sigma) \Gamma(\sigma)} \sum_{k=0}^{n} \int_{t_{k}}^{t_{k-1}} \frac{\left(\lambda-t_{k}\right)\left(\lambda-t_{k-1}\right)}{2 !} \frac{\partial^{2}}{\partial \lambda^{2}}\left[h_{3}(\lambda, e(\lambda))\right]_{y=\epsilon_{y}}\left(t_{n+1}-\lambda\right)^{\sigma-1} d \lambda .
\end{aligned}
$$

Taking this numerical method into consideration, several numerical simulations are plotted for this fractional e-cigarette smoking model. As given in [18], we choose values of the parameters $\alpha=0.01, \mu=0.1, \beta=0.3, \gamma_{1}=0.7$ and initial values $c(0)=0.5, s(0)=0.45, e(0)=0.05$.

By the above assumptions, Figure 2 shows how smokers of e-cigarettes affect non smokers and smokers of tobacco considering different values of fractional order $\sigma$. It 
seems that the non-smokers are increase and the smokers and e-cigarette smokers are decreases as the fractional order $\sigma$ is going to 1 . In addition, the existence and uniqueness of the solution of the fractional system can be seen in Figure 2 as proved in the manuscript.

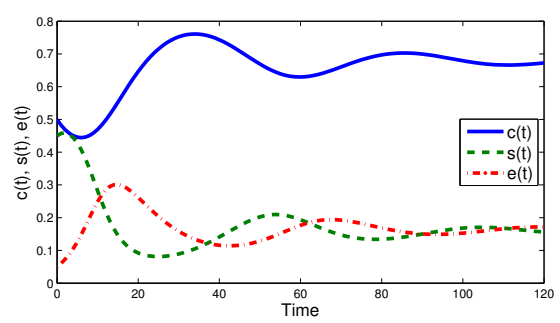

(a)

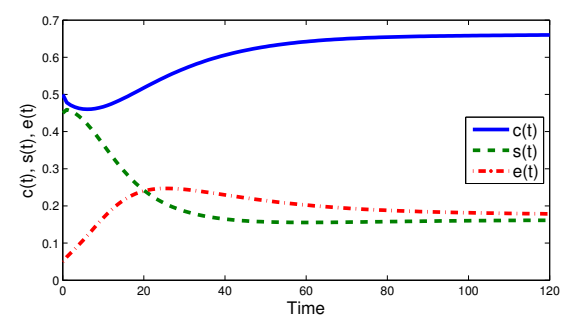

(b)

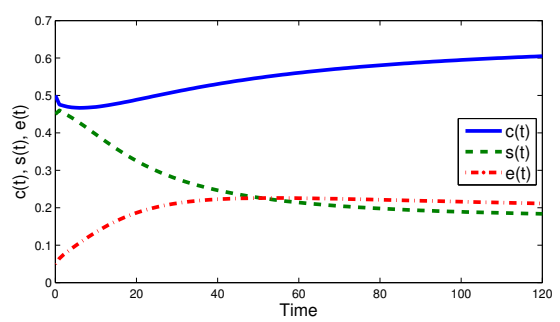

(c)

Figure 2. Numerical simulations for the Eq. (6) at (a) $\sigma=0.98$, (b) $\sigma=0.75$, (c) $\sigma=0.6$.

\section{Conclusion}

Smoking is an important public health problem in the world and has the potential to be addictive because it contains high levels of nicotine. Almost all smokers want to get rid of this problem, but quitting smoking is not really an easy task. In order to deeply investigate this matter, we examine e-cigarette smoking model by using fractional derivatives with memory properties. We give solution properties of this extended model. Then, we solve our model with numerical technique presented by Toufik and Atangana [19]. After obtaining numerical solution, we present some graphics for different values of $\sigma$ and briefly interpreted. We expect that the present study will be more advantageous in the construction of e-cigarette smoking matter benefiting from fractional derivatives. The obtained findings can lead us to do more research on this subject in the future. Especially, by using the fractional Brownian motion (fBm) the handled deterministic model can be transformed to the Stochastic Differential Equation (SDE). Hereby, it can give us more flexibility to describe the complexity and randomness of biological systems [9, 4, 27]. Furthermore, the e-cigarette smoking 
model can be modeled more realistically by using real data with uncertainty and sensitivity analysis and can be studied by considering stability analysis, fractional optimal control theory and different types of derivatives.

\section{References}

[1] Atangana A., Baleanu D., New fractional derivatives with non-local and nonsingular kernel Theory and Applications to Heat Transfer Model, Thermal Science, 20, 2016, 763-769.

[2] Atangana, A., Koca, I., Chaos in a simple nonlinear system with Atangana-Baleanu derivatives with fractional order, Chaos, Solitons \& Fractals, 89, $2016,447-454$.

[3] Evirgen F., Uçar S., Özdemir, N., System analysis of HIV infection model with CD4+T under non-singular kernel derivative, Applied Mathematics and Nonlinear Sciences, 5, 1, 2020, 139-146.

[4] Filatova D.V., Grzywaczewski M., Mathematical modeling in selected biological systems with fractional Brownian motion, 2008 Conference on Human System Interactions, IEEE, 2008, pp. 909-914.

[5] Gülşen A., Uslu B., Health hazards and complications associated with electronic cigarettes: a review, Turkish Thoracic Journal, 2020, DOI: 10.5152/TurkThoracJ.2019.180203.

[6] Gülşen A., Suicides with electronic cigarettes, General Internal Medicine, 2020, 81-88.

[7] Hung J.H., Park A., Jung I.H., Qualitative and Sensitivity Analysis of the Effect of Electronic Cigarettes on Smoking Cessation, Computational and Mathematical Methods in Medicine, 2018, 2018.

[8] Jajarmi A., Baleanu D., A new fractional analysis on the interaction of HIV with CD4+ T-cells, Chaos, Solitons \& Fractals, 113, 2018, 221-229.

[9] Jumarie G., New stochastic fractional models for Malthusian growth, the Poissonian birth process and optimal management of populations, Mathematical and Computer Modelling, 44(3-4), 2006, 231-254.

[10] Khalid M., Khan F.S., Iqbal A., Perturbation-iteration algorithm to solve fractional giving up smoking mathematical model. International Journal of Computer Applications, 142, 9, 2016.

[11] Kim J.S., Kim K., Electronic cigarette use and suicidal behaviors among adolescents, Journal of Public Health, 2019, DOI: 10.1093/pubmed/fdz086. 
[12] Koca I., Modelling the spread of Ebola virus with Atangana-Baleanu fractional operators, The European Physical Journal Plus, 133:100, 2018.

[13] Koca I., Analysis of rubella disease model with non-local and non-singular fractional derivatives, An International Journal of Optimization and Control: Theories 6 Applications, 8, 2018, 17-25.

[14] Kumar S., Kumar R., Singh J., Nisar K.S., Kumar D., An efficient numerical scheme for fractional model of HIV-1 infection of CD4+ T-cells with the effect of antiviral drug therapy, Alexandria Engineering Journal, 2020, DOI: 10.1016/j.aej.2019.12.046.

[15] Perelson A.S., Kirschner D.E., De Boer, R., Dynamics of HIV infection of CD4+ T cells, Mathematical Biosciences, 114, 1, 1993, 81-125.

[16] Shabestari P.S., Panahi S., Hatef B., Jafari S., Sprott J.C., A new chaotic model for glucose-insulin regulatory system, Chaos, Solitons $\&$ Fractals, 112, 2018, 44-51.

[17] Singh J., Kumar D., Hammouch Z., Atangana A., A fractional epidemiological model for computer viruses pertaining to a new fractional derivative, Applied Mathematics and Computation, 316, 2018, 504-515.

[18] Straughan B., E-cigarette smoking with peer pressure, Mathematical Methods in the Applied Sciences, 42, 6, 2019, 2098-2108.

[19] Toufik M., Atangana A., New numerical approximation of fractional derivative with non-local and non-singular kernel: Application to chaotic models, The European Physical Journal Plus, 2017, 132:444.

[20] Uçar S., Uçar E., Özdemir N., Hammouch Z., Mathematical analysis and numerical simulation for a smoking model with Atangana-Baleanu derivative, Chaos, Solitons \& Fractals, 118, 2019, 300-306.

[21] Uçar S., Özdemir N., Koca İ., Altun, E., Novel analysis of the fractional glucose-insulin regulatory system with non-singular kernel derivative, The European Physical Journal Plus, 135(6), 2020, 414.

[22] WHO report on the global tobacco epidemic 2019, https://apps.who.int/iris/bitstream/handle/10665/326043/9789241516204eng.pdf?ua=1, Accessed date: May 15, 2020.

[23] Tobacco Free initiative - MPOWER measures, http://www.emro.who.int/tfi/mpower/index.html, Accessed date: May 15, 2020 .

[24] Yadav A., Srivastava P.K., Kumar A., Mathematical model for smoking: Effect of determination and education, International Journal of Biomathematics, 8, 1, $2015,1550001$. 
[25] Yavuz M., Özdemir, N., Analysis of an epidemic spreading model with exponential decay law, Mathematical Sciences and Applications E-Notes, 8, 1, 2020, 142-154.

[26] Yavuz M., Yokus A., Analytical and numerical approaches to nerve impulse model of fractional-order, Numerical Methods for Partial Differential Equations, 2020, doi.org/10.1002/num.22476.

[27] Yerlikaya-Özkurt F., Vardar-Acar C., Yolcu-Okur Y., Weber G.W., Estimation of the Hurst parameter for fractional Brownian motion using the CMARS method, Journal of Computational and Applied Mathematics, 259, 2014, 843-850.

[28] Zaman G., Optimal campaign in the smoking dynamics, Computational and Mathematical Methods in Medicine, 148, 2011, 9 pages.

Received 14.06.2020, Accepted 15.12.2020 